\title{
THE LATTICE OF LEFT IDEALS IN A CENTRALIZER NEAR-RING IS DISTRIBUTIVE
}

\author{
KIRBY C. SMITH
}

\begin{abstract}
A decomposition theorem for a left ideal in a finite centralizer near-ring is established. This result is used to show that the lattice of left ideals in a finite centralizer near-ring is distributive.
\end{abstract}

1. Introduction. In the development of a density theorem for 2-primitive nearrings with identity, as presented by Betsch in [1], a key lemma for the proof of the density theorem is Lemma 2.9 of [1] due to Wielandt [6].

LEMMA (WIELANDT). Let $N$ be an arbitrary near-ring and let $B, C, D$ be $N$-submodules of some $N$-module. Then the $N$-module

$$
\Gamma=\frac{(B+D) \cap(C+D)}{(B \cap C)+D}
$$

is commutative, and for all $n \in N$ the mapping $\Gamma \rightarrow \Gamma$ defined by $\gamma \rightarrow n(\gamma)$ is an endomorphism of $(\Gamma,+)$.

An immediate consequence of Wielandt's lemma is the following found in [1].

COROLLARY. Let $N$ be a near-ring with identity such that no nonzero homomorphic image of $N$ is a ring, then the lattice of left ideals of $N$ is distributive, that is $(B+D) \cap(C+D)=(B \cap C)+D$ for any left ideals $B, C, D$ of $N$.

Thus in near-rings $N$ that satisfy the hypothesis of the corollary, the lack of elementwise left distributivity in $N$ is compensated for by a gain in the distributivity of left ideals.

It is natural to ask which near-rings have the property that their lattice of left ideals is distributive. It is the goal of this paper to show that if $N$ is a finite centralizer near-ring then the lattice of left ideals of $N$ is distributive. Since such a near-ring can have a nonzero ring as a homomorphic image (see [4]), this result does not follow from the corollary to Wielandt's lemma.

We begin by recalling the definition of a centralizer near-ring. Let $(G,+)$ be a group with identity 0 and $A$ a group of automorphisms of $G$. The centralizer near-ring determined by $G$ and $A$ is the set

$$
C(A ; G)=\{f: G \rightarrow G \mid f \alpha=\alpha f \text { for all } \alpha \in A, f(0)=0\},
$$

forming a near-ring under function addition and function composition. Centralizer near-rings arise naturally in the classification of 2-primitive near-rings [5, Chapter 4] and play a role in near-ring theory analogous to that of matrix rings in ring

Received by the editors September 15, 1981.

1980 Mathematics Subject Classification. Primary 16A76.

Key words and phrases. Centralizer, near-ring, distributive lattice.

(c) 1982 American Mathematical Society 0002-9939/81/0000-0790/\$02.00 
theory. In this paper we deal only with finite centralizer near-rings, that is $(G,+)$ is a finite group.

We now establish some concepts and notations used throughout this paper in relation to the centralizer near-ring $N=C(A ; G)$. For $v \in G$ we denote by $\operatorname{stab}(v)$ the stabilizer subgroup $\{\alpha \in A \mid \alpha v=v\}$ of $A$ and by $\theta(v)$ the $A$-orbit of $G$ containing $v$. Two orbits $\theta(w), \theta(v)$ are synonymous, written $\theta(w) \sim \theta(v)$, if there exist $w^{\prime} \in \theta(w), v^{\prime} \in \theta(v)$ with $\operatorname{stab}\left(w^{\prime}\right)=\operatorname{stab}\left(v^{\prime}\right)$. The set of all orbits of $G$ is partially ordered as follows: $\theta(w)<\theta(v)$ if and only if there exist $w^{\prime} \in \theta(w)$, $v^{\prime} \in \theta(v)$ such that $\operatorname{stab}\left(w^{\prime}\right) \supset \operatorname{stab}\left(v^{\prime}\right)$ (proper containment). We will use the notation $\theta(w) \lesssim \theta(v)$ to mean $\theta(w)<\theta(v)$ or $\theta(w) \sim \theta(v)$. Similarly the elements of $G$ are partially ordered as follows: $w<v$ if and only if $\operatorname{stab}(w) \supset \operatorname{stab}(v)$ (proper containment), and $w \sim v$ if and only if $\operatorname{stab}(w)=\operatorname{stab}(v)$. Finally $w \lesssim v$ means $\operatorname{stab}(w) \supseteq \operatorname{stab}(v)$. It is easy to see that $w \lesssim v$ if and only if there exists an element $f \in C(A ; G)$ such that $f(v)=w$, a result due to $\mathrm{G}$. Betsch (at the 1976 Oberwolfach Conference on near-rings).

Throughout this article $\theta\left(v_{1}\right), \theta\left(v_{2}\right), \ldots, \theta\left(v_{n}\right),\{0\}$ are assumed to be the $A$-orbits of the finite group $G$. The orbit representatives $v_{1}, \ldots, v_{n}$ are assumed to have the property that if $\theta\left(v_{i}\right) \lesssim \theta\left(v_{j}\right)$ then $v_{i} \lesssim v_{j}$. A function $f \in C(A ; G)$ is completely determined once its action on each $v_{i}$ is known. In analogy with matrix units in complete matrix rings we define the following special functions on $G$ which belong to $C(A ; G)$. For $i=1, \ldots, n$ let $e_{i}: G \rightarrow G$ be the identity on $\theta\left(v_{i}\right)$ and zero off $\theta\left(v_{i}\right)$. Each $e_{i}$ is idempotent and $1=e_{1}+\cdots+e_{n}$. For orbits $\theta\left(v_{i}\right), \theta\left(v_{j}\right)$ with $\theta\left(v_{i}\right) \lesssim \theta\left(v_{j}\right)$ define $e_{i j}: G \rightarrow G$ by $e_{i j}\left(v_{j}\right)=v_{i}$ and $e_{i j}$ is zero off $\theta\left(v_{j}\right)$.

2. Decomposition of left ideals. In this section we derive a decomposition theorem for left ideals $L$ in $C(A ; G)$ which will be used in the final section to prove that the left ideals of $C(A ; G)$ form a distributive lattice.

LEMMA 1. Suppose $L$ is a left ideal of $C(A ; G)$ and let $\theta\left(v_{k}\right), \theta\left(v_{j}\right)$ be orbits of $G$ under $A$ with $v_{k}<v_{j}$. If there exists an $f \in L$ such that $f\left(v_{j}\right) \in \theta\left(v_{k}\right)$ and $f\left(v_{j}\right)+v_{j} \in \theta\left(v_{k}\right)$, then $e_{j} \in L$.

ProOF. Since $e_{k} f \in L$ we may assume the range of $f$ is $\theta\left(v_{k}\right) \cup\{0\}$. Let $g=$ $e_{k}\left(f+e_{j}\right)-e_{k} e_{j}=e_{k}\left(f+e_{j}\right)$, an element in $L$. We have $g\left(v_{j}\right)=e_{k}\left(f\left(v_{j}\right)+v_{j}\right)=$ $f\left(v_{j}\right)+v_{j}$, and $g(x)=f(x)$ for $x \notin \theta\left(v_{j}\right)$. So $-f+g \in L$ and $(-f+g)\left(v_{j}\right)=$ $-f\left(v_{j}\right)+f\left(v_{j}\right)+v_{j}=v_{j},(-f+g)(x)=0, x \notin \theta\left(v_{j}\right)$. Hence $-f+g=e_{j} \in L$.

LEMMA 2. Suppose $L$ is a left ideal of $C(A ; G)$ and let $\theta\left(v_{i}\right)$ be an orbit of $G$ under $A$. If $f \in L$ is such that $f\left(v_{i}\right) \sim v_{i}$ then $e_{i} \in L$.

Proof. We may assume $f\left(v_{i}\right)=v_{i}$. For if $f\left(v_{i}\right) \in \theta\left(v_{j}\right)$ then $\theta\left(v_{j}\right) \sim \theta\left(v_{i}\right)$ and $e_{i j} \in C(A ; G)$. Also $e_{i j} f \in L$ with $e_{i j} f\left(v_{i}\right) \in \theta\left(v_{i}\right)$. Moreover some power of $e_{i j} f$ is the identity on $\theta\left(v_{i}\right)$.

As in the proof of Lemma 1 we may also assume that the range of $f$ is $\theta\left(v_{i}\right) \cup\{0\}$. Hence if $f\left(v_{k}\right) \neq 0$ for some $k \neq i$, then $f\left(v_{k}\right)=\beta_{k} v_{i}, \beta_{k} \in A$.

Finally we may assume $f$ is nonzero off $\theta\left(v_{i}\right)$, for otherwise $f=e_{i}$ and we are done. Among all such $f \in L$, select $f$ so that the number of such orbits $\theta\left(v_{k}\right)$ for which $f\left(v_{k}\right) \neq 0$ is minimal. Suppose $f\left(v_{k}\right)=\beta_{k} v_{i}, k \neq i$.

Case 1. Assume there exists a $w \in G$ such that $w \neq 0, w \lesssim v_{i}, w \notin \theta\left(v_{i}\right)$ and $v_{i}+w \notin \theta\left(v_{i}\right)$. Let $g$ be the element in $C(A ; G)$ with $g\left(v_{i}\right)=\widetilde{0}, g\left(v_{k}\right)=\beta_{k} w$ and 
$g(x)=0$ if $x \notin \theta\left(v_{i}\right) \cup \theta\left(v_{k}\right)$. Then $e_{i}(f+g)-e_{i} g \in L$ and $e_{i}(f+g)-e_{i} g=e_{i}$ due to the minimality of $f$. Hence $e_{i} \in L$ as desired.

Case 2. Assume $v_{i}+w \in \theta\left(v_{i}\right)$ for every $w$ such that $w \lesssim v_{i}, w \notin \theta\left(v_{i}\right)$. In this case we claim $\theta\left(v_{i}\right)$ is synonymous only to itself. For suppose $\theta\left(v_{i}\right) \sim \theta\left(v_{k}\right)$, yet $\theta\left(v_{i}\right) \neq \theta\left(v_{k}\right)$ where $v_{i} \sim v_{k}$. Let $\alpha_{1} v_{i}=v_{i}, \alpha_{2} v_{i}, \ldots, \alpha_{t} v_{i}$ be the distinct elements of $\theta\left(v_{i}\right)$ having the same stabilizer as $v_{i}$, that is $\alpha_{j} v_{i} \sim v_{i}, j=1,2, \ldots, t$. Then since $\theta\left(v_{i}\right) \sim \theta\left(v_{k}\right), \alpha_{1} v_{k}=v_{k}, \alpha_{2} v_{k}, \ldots, \alpha_{t} v_{k}$ are the distinct elements of $\theta\left(v_{k}\right)$ which are synonymous to $v_{i}$. By assumption $v_{i}+\alpha_{j} v_{k} \in \theta\left(v_{i}\right)$ for $j=1,2, \ldots, t$. Moreover these elements are all distinct and $v_{i}+\alpha_{j} v_{k} \sim v_{i}$ for all $j$. But none is equal to $v_{i}$, so $\theta\left(v_{i}\right)$ contains $t+1$ elements $v_{i}, v_{i}+v_{k}, \ldots, v_{i}+\alpha_{t} v_{k}$ synonymous with $v_{i}$. This contradicts $\theta\left(v_{i}\right)$ having $t$ such elements. Hence $\theta\left(v_{i}\right)$ is a unique orbit type as claimed.

We now have that if $f\left(v_{k}\right)=\beta_{k} v_{i}$ for some $k \neq i$ then $v_{i}<v_{k}$. If $\beta_{k} v_{i}+v_{k} \notin$ $\theta\left(v_{i}\right)$ then $e_{i}\left(f+e_{k}\right)-e_{i} e_{k}=e_{i}$ due to the minimality of $f$. So $e_{i} \in L$. If $\beta_{k} v_{i}+v_{k} \in \theta\left(v_{i}\right)$, then Lemma 1 applies and $e_{k} \in L$. This means $f-f e_{k}=e_{i} \in$ $L$, due to the minimality of $f$.

THEOREM 1. Let $L$ be a left ideal of $C(A ; G)$. Then for each orbit $\theta\left(v_{i}\right)$ of $G$ under $A, L e_{i} \subseteq L$.

ProOF. Select $f \in L$. If $f\left(v_{i}\right)=0$ then $f e_{i}=0 \in L$, so we may assume $f\left(v_{i}\right)=w \in \theta\left(v_{k}\right)$. We have $e_{k} f \in L$ and $e_{k} f e_{i}=f e_{i}$. Thus we may assume the range of $f$ is contained in $\theta\left(v_{k}\right) \cup\{0\}$. If $f$ is zero off $\theta\left(v_{i}\right)$ then $f e_{i}=f \in L$ and we are done. As in the proof of Lemma 2 we may reselect $f$ so that it agrees with the original function on $\theta\left(v_{i}\right)$ and is nonzero on a minimal number of orbits. Selecting $x \notin \theta\left(v_{i}\right)$ such that $f(x) \neq 0$ means $f(x)=\alpha v_{k}$ for some $\alpha \in A$. Since $w \in \theta\left(v_{k}\right)$, $x$ may be selected so that $x \gtrsim w$.

Case 1. Assume $x>w$. We have $f(x)=\alpha v_{k}$. If $f(x)+x=\alpha v_{k}+x \notin \theta\left(v_{k}\right)$, then $e_{k}\left(f+e_{x}\right)-e_{k} e_{x}=f e_{i}$ due to the minimality of $f$. So in this situation $f e_{i} \in L$. Assume now that $f(x)+x \in \theta\left(v_{k}\right)$. Let $g=e_{k}\left(f+e_{x}\right)-e_{k} e_{x}$. Then $g(x)=f(x)+x$ and $g=f$ off $\theta(x)$. We have $g \in L$ and $(-f+g)(x)=-f(x)+f(x)+x=x$ and $-g+f$ is zero off $\theta(x)$. Hence $-f+g=e_{x} \in L$. So $f-f e_{x}=f e_{i} \in L$, again using the minimality of $f$.

Case 2. Assume $x \sim w$. Then $f(x)=\alpha v_{k}$ for some $\alpha \in A$. Hence $e_{x} \in L$ by Lemma 2 and $f-f e_{x}=f e_{i} \in L$, again using the minimality of $f$.

Corollary. Let $L$ be a left ideal of $C(A ; G)$. Then $L=L e_{1} \oplus \cdots \oplus L e_{n}$.

Proof. From the theorem, $L e_{1}+\cdots+L e_{n} \subseteq L$. Also if $f \in L$ then $f=$ $f e_{1}+\cdots+f e_{n}$. Thus $L=L e_{1} \oplus \cdots \oplus L e_{n}$ since

$$
L e_{i} \cap\left(L e_{1}+\cdots+L e_{i-1}+L e_{i+1}+\cdots+L e_{n}\right)=\{0\} .
$$

3. The lattice of left ideals of $C(A ; G)$ is distributive. Let $L$ and $L^{\prime}$ be left ideals of $C(A ; G)$. From the corollary to Theorem $1, L=\sum L e_{i}$ and $L^{\prime}=\sum L^{\prime} e_{i}$. We have

(1) $L=L^{\prime}$ iff $L e_{i}=L^{\prime} e_{i}$ for every $i$,

(2) $L+L^{\prime}=\sum\left(L+L^{\prime}\right) e_{i}$,

(3) $L \cap L^{\prime}=\sum\left(L \cap L^{\prime}\right) e_{i}$. 
Now let $B=\sum B e_{i}, C=\sum C e_{i}$ and $D=\sum D e_{i}$ be left ideals of $C(A ; G)$. Using properties (2) and (3) above we have

$$
\begin{aligned}
(B+D) \cap(C+D) & =\sum\left(B e_{i}+D e_{i}\right) \cap\left(B e_{i}+D e_{i}\right) \\
& =\sum((B+D) \cap(C+D)) e_{i}, \\
(B \cap C)+D & =\sum\left(B e_{i} \cap C e_{i}\right)+D e_{i} \\
& =\sum((B \cap C)+D) e_{i} .
\end{aligned}
$$

Using property (1) we have established the following lemma.

LEMMA 3. Let $B, C$ and $D$ be left ideals of $C(A ; G)$. Then $(B+D) \cap(C+D)=$ $(B \cap C)+D$ if and only if $\left(B e_{i}+D e_{i}\right) \cap\left(C e_{i}+D e_{i}\right)=\left(B e_{i} \cap C e_{i}\right)+D e_{i}$ for $i=1, \ldots, n$.

We note that $B e_{i}, C e_{i}, D e_{i}$ are left ideals of $N=C(A ; G)$ contained in the left ideal $N e_{i}$. Lemma 3 implies that the lattice of left ideals of $N=C(A ; G)$ is distributive provided the lattice of left ideals of $N$ contained in $N e_{i}$ is distributive for $i=1, \ldots, n$.

For each $i$ let $T\left(v_{i}\right)=\left\{w \in G \mid w \lesssim v_{i}\right\}$, a subgroup of $G$. For $y \in G$ let $P\left(y ; v_{i}\right)=\left\{w \in \theta(y) \mid w \lesssim v_{i}\right\}$. The following result whose proof can be found in [3] has relevance to our problem.

THEOREM 2. Let $N=C(A ; G)$ with $v_{i} \in G^{*}, G^{*} \equiv G-\{0\}$. Then there exists a one-to-one correspondence between left ideals $L$ of $N$ contained in $N e_{i}$ and subsets $H$ of $G$ such that

(i) $H$ is a normal subgroup of $T\left(v_{i}\right)$,

(ii) $H$ is $N$-invariant,

(iii) $P\left(y ; v_{i}\right)$ is a union of cosets of $H$ for all $y \in T\left(v_{i}\right)-H$,

(iv) if $y \in T\left(v_{i}\right)-H, \alpha \in A$ such that $\alpha y-y \in H$ then $\alpha z-z \in H$ for all $z \in T\left(v_{i}\right)$ with $\operatorname{stab}(z) \supseteq \operatorname{stab}(y)$.

The correspondence mentioned in Theorem 2 is given by $L \rightarrow H_{L}$ where $H_{L}=$ $\left\{w \mid w=f\left(v_{i}\right)\right.$ for some $\left.f \in L\right\} \equiv L v_{i}$.

LEMMA 4. Suppose $L_{1}$ and $L_{2}$ are left ideals of $N=C(A ; G)$ contained in $N e_{i}$. Then either $L_{1} \subseteq L_{2}$ or $L_{2} \subseteq L_{1}$.

Proof. Suppose $L_{1}, L_{2}$ are such that $L_{1} \nsubseteq L_{2}$ and $L_{2} \nsubseteq L_{1}$. We have $L_{1} \rightarrow$ $H=L_{1} v_{i}$ and $L_{2} \rightarrow K=L_{2} v_{i}$. Since $L_{1} \& L_{2}$ then $H \& K$ and since $L_{2} \& L_{1}$ then $K \underline{Z}_{\tilde{k}} H$. Also $L_{1}+L_{2} \rightarrow H+K$. Select $\tilde{h} \in H, \tilde{k} \in K$ such that $\tilde{h}+\tilde{k} \notin H$ and $\tilde{h}+\tilde{k} \notin K$. Since $\tilde{h}+\tilde{k} \in H+K$ there exists an $f \in L_{1}+L_{2}$ such that $f\left(v_{i}\right)=\tilde{h}+\tilde{k}$. We have $f\left(v_{i}\right) \in T\left(v_{i}\right)-K$ so by Theorem 2, part (iii), $\underset{\tilde{k}}{P}\left(f\left(v_{i}\right) ; v_{i}\right)$ is a union of cosets of $K$. This means $P\left(f\left(v_{i}\right) ; v_{i}\right) \supseteq f\left(v_{i}\right)+K=h+\tilde{k}+K$ and so $\tilde{h} \in P\left(f\left(v_{i}\right) ; v_{i}\right)$.

Also $f\left(v_{i}\right) \in T\left(v_{i}\right)-H$ and by Theorem 2, part (iii), $P\left(f\left(v_{i}\right) ; v_{i}\right)$ is a union of cosets of $H$. But $\tilde{h} \in P\left(f\left(v_{i}\right) ; v_{i}\right)$, so $P\left(f\left(v_{i}\right) ; v_{i}\right) \supseteq h+H=H$. This means $0 \in P\left(f\left(v_{i}\right) ; v_{i}\right)$, a contradiction to the definition of $P\left(f\left(v_{i}\right) ; v_{i}\right)$.

THEOREM 3. The lattice of left ideals of $N=C(A ; G)$ is distributive.

ProOF. From Lemma 3 it suffices to prove that the lattice of left ideals of $N$ contained in $N e_{i}$ is distributive for each $i$. From Lemma 4 the left ideals of $N$ contained in $N e_{i}$ form a chain and hence the lattice is distributive (see [2, p. 441]). 


\section{REFERENCES}

. Betsch, Primitive near-rings, Math. Z. 130 (1973), 351-361.

. Jacobson, Basic algebra. I, Freeman, San Francisco, Calif., 1974.

. J. Maxson and K. C. Smith, Centralizer near-rings: Left ideals and 0-primitivity, Proc. Roy. ish Acad. Sect. A (to appear).

. J. Maxson, M. R. Pettet and K. C. Smith, On semisimple rings that are centralizer near-rings, acific J. Math. (to appear).

i. Pilz, Near-rings, North-Holland, Amsterdam, 1977.

[. Wielandt, Unpublished manuscripts from the years 1937-1952.

partment of Mathematics, Texas A\&M University, College Station, S 77843 